\title{
STUDIES OF CONGENITAL HEART DISEASE. I. TECHNIQUE OF VENOUS CATHETERIZATION AS A DIAGNOSTIC PROCEDURE ${ }^{1}$
}

\author{
By L. DEXTER, F. W. HAYNES, C. S. BURWELL, E. C. EPPINGER, R. E. SEIBEL, \\ AND J. M. EVANS \\ (From the Medical Clinic and the Department of Radiology, Peter Bent Brigham Hospital, \\ and the Departments of Medicine and Radiology, Harvard Medical School)
}

(Received for publication January 5, 1947)

Recent advances in the surgical treatment of congenital disorders of the heart ( 1 to 4 ) have increased the importance of accurate diagnosis. Physical and x-ray signs are not easily understood or interpreted, partly because there is relatively little systematic knowledge of the dynamics of the circulation in patients with congenital heart lesions. In the past it has been difficult to acquire such knowledge. Many of the methods of measuring cardiac output are applied with difficulty, if at all, in patients with congenital heart disease, and certain aspects of the pulmonary circulation have heretofore been almost immune from measurement.

The introduction by Forssman (5) of the cardiac catheter and its practical improvement by Cournand and Ranges (6) have offered a new opportunity for the study of congenital heart disease. By these techniques, pressures may be measured in the great veins, the right auricle and ventricle, and the pulmonary artery. Analysis of blood samples obtained from these areas and from a systemic artery permits the calculation of the cardiac output by the Fick principle. This communication describes the methods as they have been applied to patients with congenital cardiac defects. Subsequent papers will describe results obtained in controls and in patients with various congenital cardiac lesions.

\section{METHOD}

Patients with congenital heart disease are admitted to the hospital, preferably for a period of 4 days and 3 nights. Record is made of the history, physical examination, red blood cell count, hemoglobin, hematocrit, vital capacity, and usually the circulation time (cyanide or decholin) and venous pressure. $\mathrm{X}$-ray films are taken and fluoroscopic examination of the heart is carried out. The catheterization routine as developed has been de-

1 This investigation was aided in part by a grant from the John and Mary R. Markle Foundation and from the Proctor Fund of the Harvard Medical School. signed specifically for the investigation of congenital heart disease in order to assist in the diagnosis and to contribute additional information relative to the disordered circulation in the presence of the congenital abnormality. The following routine of measuring oxygen consumption, carrying out venous catheterization, and obtaining blood by arterial puncture in sequence instead of simultaneously is not the customary procedure for calculating cardiac output, but has been developed because it allows multiple sampling from each chamber. The advantages of multiple sampling will be dealt with in subsequent communications $(7,8)$.

At 7:30 on the morning of the third hospital day, the basal metabolic rate is measured and the oxygen consumption calculated. Immediately after this procedure the patient is transferred to the fluoroscopy room for venous catheterization. Although the 'technique of this procedure has been described in detail by Cournand and his associates (9), certain modifications have been introduced to meet specific problems. The patient lies on a radiolucent mat of sponge rubber, 2 inches thick, on the fluoroscopy table. This aids in maintaining comfort and, hence, in avoiding venous spasm. Ten thousand units of penicillin are administered intramuscularly before and again after catheterization even though there seems to be little risk of producmg bacterial endocarditis by this procedure. No medication other than penicillin and novocaine without adrenalin is administered. With aseptic precautions, a No. $8-\mathrm{F}$ or No. $9-\mathrm{F}$ radiopaque catheter, ${ }^{2} 100 \mathrm{~cm}$. long, with the hole at the tip and with the end curved at an angle of about $45^{\circ}$, is used. The technique of right heart catheterization as described by Cournand et al (9) has been followed closely. Once the tip has been introduced into the right auricle and the right ventricle, it is directed upward so that it is deflected from the left wall of the right ventricle, through the pulmonary valve, and into the main stem of the pulmonary artery. Since the curve of the tip of the catheter at this point is to the patient's right, it usually passes easily into the right pulmonary artery. To introduce the catheter into the left branch, it

2 This catheter can be obtained from the United States Catheter \& Instrument Corp., Glens Falls, New York. For this procedure a fairly stiff catheter has been found to be far less traumatic than a limp one. It has been our custom to heat-harden the catheter to the desired stiffness by placing it in a form for half an hour or so in a dry air oven at $120^{\circ} \mathrm{C}$. 
is withdrawn to the main stem of the pulmonary artery and is rotated in such a fashion as to turn the curved tip to the patient's left. It may then be directed into the left pulmonary artery. This rotation is sometimes difficult to accomplish since the tip of the catheter is at the end of a horseshoe bend.

Samples of blood from pulmonary "capillaries" are obtained by introducing the catheter as far out as possible into a distal ramification of one of the pulmonary arteries (see Figure 1) in such a fashion as to obstruct the vessel.
If the catheter is properly placed, fully oxygenated (arterial) blood may be withdrawn through the lumen of the catheter $(7,10)$.

Blood samples are withdrawn through the catheter under oil as described by Cournand et al (9), x-ray films are taken, and pressures with a Hamilton manometer (11) are routinely recorded at each position of the catheter. Blood samples and pressures are obtained first from the pulmonary artery, next from the right ventricle, and finally from the right auricle and superior vena cava. This

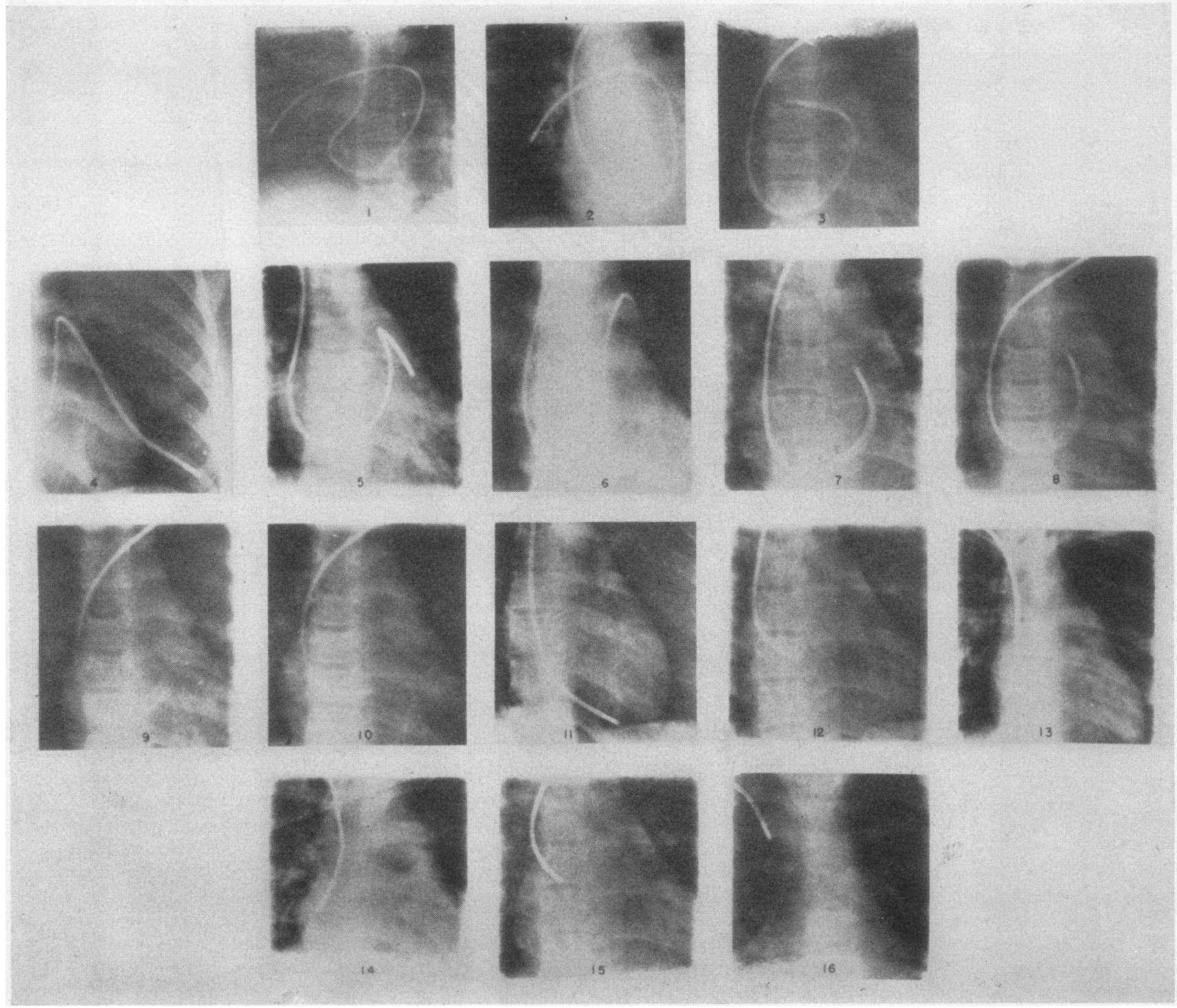

Fig. 1. X-Rays Showing the Catheter in Different Parts of the Heart and Pulmonary Artery

(1) Right and (4) left pulmonary "capillaries"; (2) and (3) right pulmonary artery; (5) and (6) left pulmonary artery; (7) main trunk of pulmonary artery; (8) right ventricle near pulmonary valve; (9) middle of right ventricle; (10) right ventricle near tricuspid valve; (11) lower part of right ventricle; (12) right auricle near tricuspid valve; (13) lower part of right auricle; (14) middle of right auricle; (15) upper part of right auricle; (16) superior vena cava. 
order of sampling is followed in order to avoid the dilemma encountered when venous spasm prevents all manipulation of the catheter except its withdrawal. In this way, multiple sampling from each chamber is ensured.

Sites of routine sampling are as follows (see Figure 1) :

1. Pulmonary artery.

a. As far out as possible (for pulmonary "capillary" blood) $(7,10)$.

b. Several centimeters beyond bifurcation.

c. Main stem.

2. Right ventricle.
a. Near pulmonary valve.
b. Mid-position.
c. Near tricuspid valve.

3. Right auricle.
a. Lower part.
b. Near tricuspid valve.
c. Upper part.

4. Superior vena cava.

While it is often not feasible or possible to obtain samples from each of these positions, at least 2 and preferably 3 samples are withdrawn from different parts of each chamber in every case.

Knowledge of the precise location of the tip of the catheter is essential for proper interpretation of data. While an approximation of the location may be made by fluoroscopic observation, it is frequently impossible to ascertain on which side of a valve the catheter tip lies. Under these circumstances, observation of the deflections of the Hamilton manometer before and after the sampling gives precise information regarding the position of the tip of the catheter since the character of the pulse wave and the pressures are quite different in pulmonary artery, right ventricle, and right auricle (7).

Following the advice of Cournand (12), we have not ligated the vein following removal of the catheter. Instead, the skin edges have been approximated with flamed adhesive and a pressure dressing with an elastic bandage has been applied. Usually, little soreness and only local thrombosis of the vein ensue. After 3 days the patient himself may remove the bandage and, in a week, the adhesive strip.

At the conclusion of venous catheterization, a sample of blood is withdrawn under oil from the femoral artery using novocaine as an anesthetic. The oxygen content of each blood sample is determined by the method of Van Slyke and Neill (13). Since at least 10 samples are taken during the procedure, duplicate determinations have been performed only on those samples exhibiting a deviation from neighboring samples.

Clotting of samples is at times troublesome, especially in polycythemic patients. This has been consistently avoidable by injecting about $2 \mathrm{ml}$. of a 0.03 per cent solution of heparin through the catheter just before sampling.

Oxygen capacity is determined routinely on the arterial and at least one of the venous samples by the method of Van Slyke and Neill (13). Ordinarily the oxygen capacity of different blood samples is essentially the same. The capacity may vary considerably, however, especially in samples of blood with a high hematocrit. Hemoglobin concentrations are, therefore, determined photocolorimetrically on all samples, and those at variance are then corrected by determining the oxygen capacity. Variations in oxygen capacity may thus be detected and the corresponding contents corrected.

\section{DISCUSSION}

The procedure described in this report has been formulated with the specific purpose of studying the cardiovascular dynamics in congenital heart disease and of applying the knowledge so obtained to diagnosis.

Calculation of blood flow: The most accurate method at present available for the estimation of cardiac output in man is based on the principle outlined by Fick (14) in 1870. Some of us (15) applied this principle in a group of patients with patent ductus arteriosus in 1941. Since its application in patients with abnormal cardiac communications presents special problems, it seems appropriate to restate the principle and discuss its application in patients with this and other types of congenital heart disease which the catheter method has enabled us to investigate.

Fick (14) derived an equation for the calculation of cardiac output which may be restated as follows :

\section{Cardiac output (1. per min.) \\ Oxygen intake (ml. per min.) $=\overline{\text { Arteriovenous oxygen difference (ml. per 1.) }}$}

Similar data derived from determinations of carbon dioxide can be used. Carbon dioxide data have been omitted, however, since the results of these determinations are variable (16). In normal individuals without shunts between cardiac chambers, the calculation is based on the determination of oxygen consumption, oxygen content of arterial blood, and oxygen content of mixed venous blood. The first two determinations are easily obtained. The procurement of well-mixed venous blood is more difficult. In normal individuals, samples from the right auricle and right ventricle may show considerable variation, whereas those taken from the proximal parts of the pulmonary artery show minimal variation and represent acceptable values for mixed venous blood (7). 
In patients with congenital heart disease, as will be shown (8), it is frequently impossible to obtain samples of blood that are adequately mixed if these samples are taken near a shunt. Although this detracts from the accuracy of the estimation, nevertheless reasonable approximations of flow through the various chambers may be obtained.

To estimate the blood flow through a shunt, the peripheral blood flow and pulmonary blood flow must be calculated separately. The peripheral blood flow may be calculated in the usual fashion except that the sample of mixed venous blood must be obtained proximal to the shunt. For example, in cases with patent ductus arteriosus, it must be obtained by multiple sampling from the right ventricle; in cases with ventricular septal defect, from the right auricle; and in cases of auricular defect, from the vena cava. Since true mixing becomes progressively poorer in this sequence, errors in the application of the Fick principle of blood flow become greater.

Determination of the pulmonary artery blood flow necessitates measurement of the oxygen content of pulmonary arterial and of pulmonary venous blood. Normally, blood in the main branches of the pulmonary artery is completely mixed. In our limited experience, this is also true in most cases of auricular septal defect. In ventricular septal defect and in patent ductus arteriosus, mixing may be less complete. In these cases, multiple sampling in the pulmonary artery usually yields a representative value from which the calculation of pulmonary blood flow can be made. The oxygen content of pulmonary venous blood is most easily obtained in individuals with a normal circulation and in those with left-to-right shunts by determining the oxygen content of systemic arterial blood since these two are identical. In individuals with right-to-left shunts (cyanotic group), the oxygen content of pulmonary venous blood can be obtained by measuring the oxygen content of pulmonary "capillary" blood $(7,10)$. Failing this, a value of 95 to 98 per cent saturation must be assumed. This assumption is admittedly open to criticism, especially when pulmonary disease with imperfect oxygen diffusion exists.

The catheter technique does not afford information relative to the collateral circulation between systemic arteries and the pulmonary artery such as sometimes occurs in patients, especially with pulmonary stenosis, if these collateral channels deliver blood to the pulmonary artery beyond the point where catheter samples are obtained. Bing (17) has utilized a combination of the catheter technique and respiratory methods for calculating the magnitude of blood flow through the collateral circulation in these individuals.

The volume of flow through single, onedirectional shunts may most easily be estimated by calculating the difference between the pulmonary and peripheral flows. If the shunt is in both directions, as not infrequently occurs, the following formulae, ${ }^{3}$ derived by Dr. S. Howard Armstrong, Jr., may be used:

${ }^{3}$ Let pulmonary artery inflow $=a \mathrm{ml}$. per min. (Determined by Fick equation from pulmonary artery and left auricle oxygen saturations and oxygen consumption).

Let oxygen saturation vena cava inflow $=b$ per cent.

Let oxygen saturation pulmonary vein outflow $=c$ per cent.

Let oxygen saturation pulmonary artery inflow $=d$ per cent. Now pulmonary artery inflow has 2 components:

(1) from vena cava, $x \mathrm{ml}$. per min.,

(2) from shunt, left-to-right, $y \mathrm{ml}$. per min. Therefore

Solving for $y$, from (2)

$$
\begin{gathered}
x+y=a, \\
\frac{b x+c y}{x+y}=d .
\end{gathered}
$$

$$
\begin{gathered}
(b-d) x+(c-d) y=0 \\
x=\frac{(d-c) y}{(b-d)}
\end{gathered}
$$

and from (1)

$$
y=\frac{a}{1+\frac{(d-c)}{(b-d)}}=a \frac{(b-d)}{(b-c)} \mathrm{ml} \text {. per min. }
$$

Now if this is the amount of pulmonary vein blood shunting from left to right and out the pulmonary artery, the remainder must go out the aorta. The other component of aortic flow will shunt from right to left and have the same saturation as right auricular blood, and must equal in amount the return from the vena cava less that which has gone through the pulmonary artery. The total caval return and aortic outflow will be assumed to be equal during measurements. Therefore, for total aortic output, $u$ (in ml. per min.), the 2 components are:

(1) from shunt right to left, $v \mathrm{ml}$. per min.,

(2) from the pulmonary veins,

$$
e=a\left(1-\frac{b-d}{b-c}\right)=a\left(\frac{d-c}{b-c}\right)
$$

Therefore, $u=v+e$.

Now let $f$ per cent be the oxygen saturation of the peripheral arterial blood. Then

Solving (4) for $v$,

$$
f=\frac{b v+c e}{v+e} .
$$

$$
\begin{aligned}
(f-b) v & =(c-f) \dot{e} \\
v & =\frac{(c-f)}{(f-b)} e .
\end{aligned}
$$


Where $v=$ right-to-left shunt (1. per min.), $y=$ left-to-right shunt (1. per min.), $a=$ pulmonary blood flow (1. per min.), $b=$ oxygen content (ml. per 1.) of mixed venous blood proximal to the shunt (venae cavae, right auricle, or right ventricle), $c=$ oxygen content ( $\mathrm{ml}$. per 1.) of pulmonary venous blood, $d=$ oxygen content ( $\mathrm{ml}$. per 1.) of pulmonary arterial blood, and $f=$ oxygen content (ml. per 1.) of systemic arterial blood, then

$$
y=a \frac{(b-d)}{(b-c)} \quad v=a \frac{(c-f)(d-c)}{(f-b)(b-c)} .
$$

When 2 shunts in the same direction are present in the same heart (e.g., auricular and ventricular septal defect with left-to-right shunts), calculation of the approximate sum total of the shunts is possible, whereas it is not feasible to attempt much more than a guess at the amount of each shunt due to the impossibility of obtaining mixing between the chambers.

All of these difficulties indicate that the Fick principle, when applied to congenital heart disease, gives an estimation of blood flow which is at best an approximation. It is useful to make the calculation, but the assumptions on which such calculations are based must be clearly borne in mind.

Blood pressures: Mean pressures are easily recorded through the venous catheter by means of a simple saline manometer of the venous pressure type. Considerably more information may be obtained with the optical manometer of Hamilton (11). We have experienced difficulty in obtaining suitable pulse wave tracings from the pulmonary artery and from random positions in the right ventricle. With care, good right ventricular pulse wave tracings can be obtained. Artefacts are often

Thus

$$
u=e\left(1+\frac{(c-f)}{(f-b)}\right)=a \frac{(d-c)(c-b)}{(b-c)(f-b)},
$$

an expression completely defined in terms of our original quantities measured.

Simplifying algebraically:

Left-to-right shunt $=a \frac{(b-d)}{(b-c)}$.

Note that if $(b-d)=0$, i.e., mixed venous and pulmonary artery blood have same saturation, this means negligible left-to-right shunt.

Right-to-left shunt $=a\left(\frac{c-f}{f-b}\right)\left(\frac{d-c}{b-c}\right)$.

Note that if $(c-f)=0$, i.e., left auricle and artery blood have same saturation, this means negligible right-to-left shunt. set up, apparently by the motion of the catheter within the heart. The natural frequency of the membrane falls from about 170 vibrations per second when recorded through a needle and lead tubing to about 30 vibrations per second when recorded through the catheter and lead tubing. The systolic and diastolic pressures seem to be accurate in that systolic and diastolic pressures in the right ventricle of dogs have been identical when recorded through the catheter and through a No. 18 needle inserted through the chest wall directly into the right ventricle.

The peak of the pressure curve in both pulmonary artery and right ventricle is taken as the systolic pressure, and the level just before the systolic rise is taken as the diastolic pressure. The right auricular pressure is recorded as mean pressure.

The location of the proper level of zero pressure is still controversial since it is different for right auricle, right ventricle, and pulmonary artery. It likewise varies with systole and diastole. Until a standard reference level has been agreed upon, we have chosen to use the level described by one of us (18), which is $10 \mathrm{~cm}$. anterior to the skin of the back with the subject in the supine position. In addition, the anteroposterior diameter of the chest between the sixth thoracic spine and the sternum at the level of the second rib has been recorded with an obstetrical pelvimeter. The pressures, therefore, may be easily recalculated if it is desired to use other zero points.

It has been customary to obtain pressure tracings in the pulmonary artery, right ventricle, and right auricle. A continuous pressure tracing is usually obtained as the catheter is slowly withdrawn from pulmonary artery to right ventricle and from right ventricle to right auricle. In this way comparable pressures are recorded. If the double-lumen catheter of Cournand, Bloomfield, and Lauson (19) is used, pressures may be obtained from 2 chambers simultaneously.

Complications of venous catheterization: That venous catheterization is a benign procedure even in fairly ill patients has been affirmed repeatedly $(20,21,22)$, and our experience confirms this. Two sources of mild discomfort have been observed occasionally during venous catheterization. The first is venous spasm, which in our experi- 
ence has been almost uniformly associated with bodily discomfort but has not derived from apprehension. Every effort is made to prevent discomfort. The site of the incision is injected with novocaine about every 10 minutes, the fluoroscope table is covered with a 2 -inch sponge rubber mat, pillows are used judiciously for support of the head and shoulders, and the whole procedure is dispatched as quickly as possible (usually about 45 minutes). When venous spasm occurs, it has rarely been possible to relieve it except by removing the catheter from the vein for several minutes.

Ventricular extrasystoles may at times be a source of discomfort to the patient (9). In 27 of 42 patients, irregularity of the heart was shown to occur during venous catheterization. This was demonstrated by electrocardiogram, by pulse tracing, or by palpation. Only 14 of the 27 patients had symptoms referable to this irregularity, and in only 1 were the symptoms of sufficient severity to interrupt the procedure. Five of 12 patients who had electrocardiograms recorded at frequent intervals developed premature ventricular beats. The ventricular extrasystoles are produced especially when the catheter touches the wall adjacent to the tricuspid valve. They usually disappear when the tip of the catheter is in the ventricle, but at times the irregularity persists, apparently due to movement of the catheter with each heartbeat with consequent stimulation of the region of the tricuspid valve. Two patients developed transient auricular fibrillation which subsided spontaneously in the course of half an hour.

In obtaining pulmonary "capillary" blood, the catheter is introduced into the distal portion of a branch of the pulmonary artery so as to obstruct the vessel. It remains in this position for only a few minutes. In no case has there been any change in pulse and respiratory rates and no patient has had untoward symptoms. In dogs a main lobar branch of the pulmonary artery must be obstructed for approximately 1 hour before $x$-ray and pathological evidence of congestion or infarction appears (23). The vessels occluded by a No. 9-F catheter are roughly $3 \mathrm{~mm}$. in diameter. Since there is a slow drip of physiological saline through the catheter, it seems doubtful whether infarction would occur for many hours.

Auscultation and phonocardiography have re- vealed no demonstrable changes in the heart sounds or production of murmurs as the catheter passes through either the tricuspid or pulmonary valve. In one patient with pronounced pulmonary stenosis, a catheter about $3 \mathrm{~mm}$. in diameter was introduced past the valve into the pulmonary artery without symptoms. Following a subsequent operation the patient expired, and at autopsy the pulmonary valve orifice was found to measure only $5 \mathrm{~mm}$. in diameter. Cournand, Bloomfield, and Lauson (19) recorded pressure tracings simultaneously in right auricle and right ventricle and concluded that there was no indication of tricuspid insufficiency as a result of inserting the catheter through the tricuspid valve. It seems doubtful, therefore, that venous catheterization produces a functional stenosis or insufficiency of the tricuspid or pulmonary valve under a rather wide range of pathological conditions.

Pathological examination of the endothelium, valve leaflets, chordae tendinae, and papillary muscles in dogs purposely exposed to traumatic venous catheterization of the right auricle, right ventricle, and pulmonary artery has revealed no recognizable pathological lesions resulting from this procedure (24). No evidence of pulmonary embolism from clot formation around the catheter was observed. Post-mortem examinations have been performed on 10 of our patients on whom venous catheterization had previously been performed. In no case was death directly or indirectly attributable to the procedure. In no instance were there any demonstrable lesions of the veins, heart, pulmonary artery, or lung attributable to the passage of the catheter.

\section{SUM MARY}

1. The venous catheter technique of Cournand and Ranges (6) has been applied to the diagnosis and study of the hemodynamics of congenital heart disease.

2. Oxygen consumption is measured, and pressures and blood samples (for the oxygen content) are obtained from various parts of the pulmonary artery, right ventricle, right auricle, and from the vena cava and the femoral artery.

3. Details of the procedure are described, and the Fick principle for the calculation of blood flow in patients with congenital heart disease is discussed. 


\section{BIBLIOGRAPHY}

1. Gross, R. E., and Hubbard, J. P., Surgical ligation of a patent ductus arteriosus. Report of first successful case. J. A. M. A., 1939, 112, 729.

2. Blalock, A., and Taussig, H. B., Surgical treatment of malformations of the heart. J. A. M. A., 1945, 128, 189.

3. Crafoord, C., and Nylin, G., Congenital coarctation of aorta and its surgical treatment. J. Thoracic Surg., 1945, 14, 347.

4. Gross, R. E., and Hufnagel, C. A., Coarctation of the aorta. Experimental studies regarding its surgical correction. New England J. Med., 1945, 233, 287.

5. Forssman, W., Die Sondierung der rechten Herzens. Klin. Wchnschr., 1929, 8, 2085.

6. Cournand, A., and Ranges, H. A., Catheterization of right auricle. Proc. Soc. Exper. Biol. \& Med., 1941, 46, 462.

7. Dexter, L., Haynes, F. W., Burwell, C. S., Eppinger, E. C., Sagerson, R. P., and Evans, J. M., Studies of congenital heart disease. II. The pressure and oxygen content of blood in the right auricle, right ventricle, and pulmonary artery in control patients, with observations on the oxygen saturation and source of pulmonary "capillary" blood. J. Clin. Invest., 1947, 26, 554.

8. Dexter, L., Haynes, F. W., Burwell, C. S., Eppinger, E. C., Sosman, M. C., and Evans, J. M., Studies of congenital heart disease. III. Venous catheterization as a diagnostic aid in patent ductus arteriosus, tetralogy of Fallot, ventricular septal defect, and auricular septal defect. J. Clin. Invest., 1947, 26, 561.

9. Cournand, A., Riley, R. L., Breed, E. S., Baldwin, E. deF., and Richards, D. W., Jr., Measurement of cardiac output in man using the technique of catheterization of the right auricle or ventricle. J. Clin. Invest., 1945, 24, 106.

10. Dexter, L., Burwell, C. S., Haynes, F. W., and Seibel, R. E., Oxygen content of pulmonary "capillary" blood in unanesthetized human beings. J. Clin.' Invest., 1946, 25, 913.

11. Hamilton, W. F., Brewer, G., and Brotman, I., Pressure pulse contours in the intact animal. I. Ana-
- lytical description of a high frequency manometer.

Am. J. Physiol., 1934, 107, 427.

12. Cournand, A., Personal communication, 1945.

13. Peters, J. P., and Van Slyke, D. D., Quantitative Clinical Chemistry, Vol. 2, Methods. Williams \& Wilkins, Baltimore, 1943.

14. Fick, A., Ueber die Messung des Blutquantums in den Herzventrikeln. Verhandl. d. physical. med. gesellsch. z. Würzburg, 1870, 2, 16.

15. Eppinger, E. C., Burwell, C. S., and Gross, R. E., The effects of the patent ductus arteriosus on the circulation. J. Clin. Invest., 1941, 20, 127.

16. Cournand, A., Measurement of the cardiac output in man using the right heart catheterization. Description of technique, discussion of validity and of place in the study of the circulation. Federation Proc., 1945, 4, 207.

17. Bing, R. J., Vandam, L. D., and Gray, F. D., Jr., Physiological studies in congenital heart disease. I. Procedures. Bull. Johns Hopkins Hosp., 1947, 80, 107.

18. Lyons, R. H., Kennedy, J. A., and Burwell, C. S., Measurement of venous pressure by direct method. Am. Heart J., 1938, 16, 675.

19. Cournand, A., Bloomfield, R. A., and Lauson, H. D., Double lumen catheter for intravenous and intracardiac blood sampling and pressure recording. Proc. Soc. Exper. Biol. \& Med., 1945, 60, 73.

20. Cournand, A., Riley, R. L., Bradley, S. E., Breed, E. S., Noble, R. P., Lauson, H. D., Gregersen, M. I., and Richards, D. W., Studies on the circulation in clinical shock. Surgery, 1943, 13, 964.

21. McMichael, J., and Sharpey-Schafer, E. P., Cardiac output in man by direct Fick method; effects of posture, venous pressure change, atropine, and adrenaline. Brit. Heart J., 1944, 6, 33.

22. Brannon, E. S., Weens, H. S., and Warren, J. V., Atrial septal defect. Study of hemodynamics by the technique of right heart catheterization. Am. J. M. Sc., 1945, 210, 480.

23. Kinney, T. D., Haynes, F. W., and Dexter, L., Unpublished observations, 1946.

24. Kinney, T. D., Haynes, F. W., and Dexter, L., Experimental production of pulmonary embolism by the use of a venous catheter. J. Lab. \& Clin. Med., 1945, 30, 1013. 